\title{
Growth differences and genetic parameter estimates of 15 teak (Tectona grandis L.f.) genotypes of various ages clonally propagated by microcuttings and planted under humid tropical conditions
}

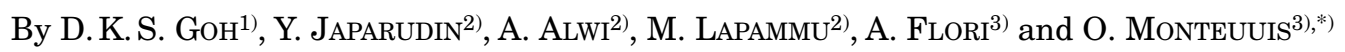

(Received $30^{\text {th }}$ November 2012)

\begin{abstract}
Fifteen clones of teak (Tectona grandis) produced by micropropagation from 0.5 to more than 60 yr-old selected ortets were established in a clonal test in Sabah (East Malaysia) under $2500 \mathrm{~mm}$ of annual rainfall to compare their growth performances during the first 7 years of development. Field establishment was good with average mortality less than $10 \%$. The clones developed rapidly true-to-type with significant between-clone differences in growth. Ranges of clone means were 13.6 to $19.3 \mathrm{~m}$ in height, 16.3 to $23.4 \mathrm{~cm}$ in diameter at breast height (DBH) and 129 to $264 \mathrm{dm}^{3}$ in volume. Broad sense heritability estimates for these growth traits were lower overall for single trees $\left(\mathrm{H}^{2} \mathrm{i}\right)$ than for clone means $\left(\mathrm{H}^{2} \mathrm{c}\right)\left(\mathrm{H}^{2} \mathrm{i} \leq 0.257 \mathrm{vs} \mathrm{H}^{2} \mathrm{c} \leq 0.634\right.$ for height, $\mathrm{H}^{2} \mathrm{i} \leq 0.120$ vs $\mathrm{H}^{2} \mathrm{c} \leq 0.383$ for $\mathrm{DBH}$ and $\mathrm{H}^{2} \mathrm{i} \leq 0.125$ vs $\mathrm{H}^{2} \mathrm{c} \leq 0.364$ for volume). The highest genetic gain that could be expected from the best three clones out of the fifteen compared was at age 2 for height $(+0.66 \mathrm{~m}$, or $+11.7 \%)$, and age 3 for $\mathrm{DBH}(+0.87 \mathrm{~cm}$, or $+10.4 \%)$ and volume $\left(+4.65 \mathrm{dm}^{3}\right.$, or $\left.+15.7 \%\right)$. Age-related phenotypic correlation values were reliably $(\mathrm{P}<0.0001)$ higher and more consistent for $\mathrm{DBH}\left(\mathrm{r}_{\mathrm{p}} \geq 0.61\right)$ than for height $\left(0.37 \leq \mathrm{r}_{\mathrm{P}} \leq 0.69\right)$, or than between $\mathrm{DBH}$ and height, except for height at $3\left(0.51 \leq \mathrm{r}_{\mathrm{P}} \leq 0.63\right)$ and $6\left(0.55 \leq \mathrm{r}_{\mathrm{p}} \leq\right.$ $0.69)$ years. Height and $\mathrm{DBH}$ were moderately to highly genetically correlated $\left(0.54 \leq \mathrm{r}_{\mathrm{G}} \leq 0.90\right)$.
\end{abstract}

Key words: broad sense heritability, clone, genetic correlations, genetic gain, growth, maturation, rejuvenation, vegetative propagation.

\section{Introduction}

Tectona grandis Linn. f., commonly known as teak, is a large arborescent species belonging to the Verbenaceae family. It is indigenous to India, Laos, Myanmar (exBurma) and Thailand (TewARI, 1992). This species remains the most prized high value timber due to the outstanding properties of its wood, with special mention for durability and aesthetic features (KADAMBI, 1972;

1) YSG Biotech Sdn Bhd, Yayasan Sabah Group, Voluntary Association Complex, Mile 21/2, off Tuaran Road, P.O.Box 11623, 88817 Kota Kinabalu, Sabah, Malaysia.

2) Sabah Softwoods Sdn Bhd, KM 8, off Sin San Road, P.O. Box 60966, 91019 Tawau, Sabah, Malaysia.

3) CIRAD-BIOS Department - UMR AGAP, TA A-108/03 Avenue Agropolis, F-34398 Montpellier, Cedex 5 France.

*) Corresponding author: Olivier MonteuUis. CIRAD-BIOS, UMR AGAP, TA A-108/03 - Avenue Agropolis, F-34398 Montpellier cedex 5 - FRANCE. Tel: 33 (0)4 67617121 , Fax: 33 (0)4 6761 5605. E-Mail: olivier.monteuuis@cirad.fr
FAO, 2009). This attractiveness has spurred its introduction for timber production outside its native range in several tropical countries of Asia, starting with Indonesia some 4 to 6 centuries ago (SISWAMARTANA, 2000; VERHAEGEN et al., 2010), then Africa and Latin America (BALL et al., 2000). The list of countries embarking on teak industrial plantations has rapidly expanded lately under private investors eager to supply the demand for high grade timber, which continues to increase in the wake of declining supplies from natural stands (FAO, 2009; Kollert and Cherubini, 2012). Teak planting activities have recently been promoted by the possibility to produce and plant clones which, when soundly selected and deployed, appear to be far superior in timber yield, quality, uniformity and reduction of rotation length to seedlings (GoH and MonTEuUIS, 2005; SMIT et al., 2011; GoH and MonTeuUIS, 2012). Protocols for efficiently mass cloning selected plus trees of any age by cuttings, shoot apical meristems or microcuttings were developed in Sabah in the early 1990s (MoNTEUUIS, 1995; MonteuUis et al., 1995; MonteuUIs et al., 1998). Different clones, some of them deriving from more than 60 year-old ortets, were produced and initially field planted in Sabah to assess their conformity to parental phenotype (GoH and MonTEUUIS, 2005; GoH et al., 2007). They were later dispatched in great quantities to various oversea countries for testing under different environmental conditions and used for the development of industrial clonal plantations (GoH et al., 2007; GoH and MonTEuUIS, 2012). In spite of this success, published information on growth performances and genetic parameters of these clones in the tropical humid conditions of Sabah where they were selected are still lacking. It is therefore timely to report on this subject to increase the limited knowledge of teak clones produced by adventitious rooting (SISWAMARTANA and WibOWO, 2005; CALlister and Collins, 2008; WARDANI et al., 2009; SOlÓRZANo NARANJO et al., 2012).

\section{Materials and Methods \\ Plant material}

The clones compared derived from 15 Tectona grandis mother trees of various estimated ages and origins, as detailed in Table 1. These ortets were selected on their superior phenotypic traits or genetic background for the younger ones, and were clonally micropropagated in vitro by axillary budding (MoNTEUUIS et al., 1998). Rooting of the microshoots produced in vitro occurred within 
Table 1. - Characteristics of the 15 teak mother trees (ortets) from which the clones were derived.

\begin{tabular}{|c|c|c|c|}
\hline $\begin{array}{l}\text { Clone } \\
\text { name }\end{array}$ & Location of the ortets & $\mathrm{Age}^{\star}$ & Additional information \\
\hline Sl1 & $\begin{array}{l}\text { Luasong Forestry Center, , Tawau } \\
\text { district, Sabah, East Malaysia }\end{array}$ & 4 yrs & $\begin{array}{c}\text { Derived from seeds from Solomon islands (seed } \\
\text { source);presumed natural provenance: Myanmar, ex } \\
\text { Burma (Goh and Monteuuis 2009, 2012) }\end{array}$ \\
\hline $\mathrm{SI} 2$ & $\begin{array}{l}\text { Luasong Forestry Center, , Tawau } \\
\text { district, Sabah, East Malaysia }\end{array}$ & 4 yrs & $\begin{array}{l}\text { Derived from seeds from Solomon islands (seed } \\
\text { source); presumed natural provenance: Myanmar, ex } \\
\text { Burma (Goh and Monteuuis 2009, 2012) }\end{array}$ \\
\hline $\mathrm{SI} 3$ & $\begin{array}{l}\text { Luasong Forestry Center, , Tawau } \\
\text { district, Sabah, East Malaysia }\end{array}$ & 4 yrs & $\begin{array}{l}\text { Derived from seeds from Solomon islands (seed } \\
\text { source);presumed natural provenance: Myanmar, ex } \\
\text { Burma \{Goh and Monteuuis 2009, 2012) }\end{array}$ \\
\hline $\mathrm{SI} 4$ & $\begin{array}{l}\text { Luasong Forestry Center, , Tawau } \\
\text { district, Sabah, East Malaysia }\end{array}$ & 4 yrs & $\begin{array}{l}\text { Derived from seeds from Solomon islands (seed } \\
\text { source); presumed natural provenance: Myanmar, ex } \\
\text { Burma (Goh and Monteuuis 2009, 2012) }\end{array}$ \\
\hline Sl6 & $\begin{array}{l}\text { Luasong Forestry Center, , Tawau } \\
\text { district, Sabah, East Malaysia }\end{array}$ & 4 yrs & $\begin{array}{l}\text { Derived from seeds from Solomon islands (seed } \\
\text { source);presumed natural provenance: Myanmar, ex } \\
\text { Burma (Goh and Monteuuis 2009, 2012) }\end{array}$ \\
\hline SI7 & $\begin{array}{l}\text { Luasong Forestry Center, , Tawau } \\
\text { district, Sabah, East Malaysia }\end{array}$ & 4 yrs & $\begin{array}{l}\text { Derived from seeds from Solomon islands (seed } \\
\text { source); presumed natural provenance: Myanmar, ex } \\
\text { Burma (Goh and Monteuuis } 2009,2012 \text { ) }\end{array}$ \\
\hline SI8 & $\begin{array}{l}\text { Luasong Forestry Center, , Tawau } \\
\text { district, Sabah, East Malaysia }\end{array}$ & 4 yrs & $\begin{array}{c}\text { Derived from seeds from Solomon islands (seed } \\
\text { source);presumed natural provenance: Myanmar, ex } \\
\text { Burma (Goh and Monteuuis } 2009,2012 \text { ) }\end{array}$ \\
\hline MBO & $\begin{array}{l}\text { Luasong Forestry Center, Tawau } \\
\text { district, Sabah, East Malaysia }\end{array}$ & $\begin{array}{c}40 \text { yrs or } \\
\text { more }\end{array}$ & $\begin{array}{c}\text { Senescing tree which died } 3 \text { yrs later (Goh and } \\
\text { Monteuuis 2009, 2012) }\end{array}$ \\
\hline DG1 & $\begin{array}{l}\text { Luasong Forestry Center, Tawau } \\
\text { district, Sabah, East Malaysia }\end{array}$ & 6 months & Germinated seed from Thailand \\
\hline DG3 & $\begin{array}{l}\text { Luasong Forestry Center, Tawau } \\
\text { district, Sabah, East Malaysia }\end{array}$ & 6 months & Germinated seed from Thailand \\
\hline MA106 & $\begin{array}{l}\text { Mata Ayer, state of Perlis, } \\
\text { West Malaysia }\end{array}$ & $40-45 \mathrm{yrs}$ & $\begin{array}{l}\text { Situation: } 75^{\circ} 29^{\prime} \text { E. Origin uncertain } \\
\text { (Krishnapillay and Abdul Razak1999) }\end{array}$ \\
\hline JA16 & $\begin{array}{c}\text { Jalan Apas, Tawau district, Sabah, } \\
\text { East Malaysia }\end{array}$ & $27 \mathrm{yrs}$ & $\begin{array}{l}\text { Derived from seeds imported from Trinidad } \\
\text { (Lapongan 2000) }\end{array}$ \\
\hline KM29 & $\begin{array}{l}\text { Bandau, Kota Marudu district, } \\
\text { Sabah, East Malaysia }\end{array}$ & $64-67$ yrs & $\begin{array}{l}\text { Planted by the Dutch Tobacco Company in } 1926 \text { or } \\
1929 \text { (Lapongan 2000) }\end{array}$ \\
\hline KM41 & $\begin{array}{l}\text { Bandau, Kota Marudu district, } \\
\text { Sabah, East Malaysia }\end{array}$ & $64-67$ yrs & $\begin{array}{l}\text { Planted by the Dutch Tobacco Company in } 1926 \text { or } \\
1929 \text { (Lapongan 2000) }\end{array}$ \\
\hline KM44 & $\begin{array}{l}\text { Bandau, Kota Marudu district, } \\
\text { Sabah. East Malaysia }\end{array}$ & $64-67 \mathrm{yrs}$ & $\begin{array}{l}\text { Planted by the Dutch Tobacco Company in } 1926 \text { or } \\
1929 \text { (Lapongan 2000) }\end{array}$ \\
\hline
\end{tabular}

* Presumed age of the ortets at the time of explant collection for in vitro cloning.

3 weeks with more than $95 \%$ success in nursery conditions, using wet sand as rooting substrate, under $50 \%$ shade and appropriate mist-system, as described in BONAL and MonteuUIS (1997). The rooted microshoots, measuring 4-6 cm in height, were then potted individually in $10 \times 15 \mathrm{~cm}$ black plastic bags filled with clayey local top soil mixed with sand for better drainage to be acclimatized. After a raising period of 3 months, the plants reached an average stem height of $20 \mathrm{~cm}$ and were thereafter field planted.

\section{Trial layout and tree sampling}

The trial was set up in January 2002 on a piece of land named 96G in the plantation area of Sabah Softwood Sdn Bhd (SSSB), Brumas, at $60 \mathrm{~km}$ west from Tawau, Sabah, East Malaysia (Lat $4^{\circ} 35^{\prime} \mathrm{N}$, Long $\left.117^{\circ} 40^{\prime} \mathrm{E}\right)$. The planting site, whose features are detailed in Table 2, was rather hilly and prepared by ripping and mounding just before planting.
A randomized complete block design with four contiguous replications was used .Plots comprised two rows each of 30 plants of the 15 clones. Spacing was $3 \times 3 \mathrm{~m}$ which resulted in a total net area of $3 \times 3 \times 30 \times 2 \times 15 \times$ $4=3.24$ ha. Only the $11^{\text {th }}$ to $20^{\text {th }}$ plants of each row were assessed, corresponding to 80 plants per clone in all.

\section{Traits recorded and statistical analyses}

The quantitative data recorded were:

1. mortality rate "M", determined as the numbers of dead trees as a percentage of the total number of trees initially planted.

2. total tree height " $\mathrm{H}$ " (in $\mathrm{m}$ ), measured (in $\mathrm{dm}$ ) with a graduated pole and then with a clinometer when trees became too tall, one (H1), two (H2), three (H3), four (H4), six (H6) and 7 (H7) years after planting.

3. diameter at breast height or " $\mathrm{DBH}$ " (in $\mathrm{cm}$ ), converted from the girth measured with a tape at about 
$1.30 \mathrm{~m}$ above soil level, three (D3), four (D4), six (D6) and seven (D7) years after planting.

4. bole volume "V" (in $\mathrm{dm}^{3}$ ), calculated three (V3), four (V4), six (V6) and seven (V7) years after planting by using the following formula:

$$
\begin{aligned}
\mathrm{V}= & \left(\left(\pi \times(\mathrm{DBH} / 2)^{2} \times 1.3\right)+\left(\pi \times(\mathrm{DBH} / 2)^{2}\right.\right. \\
& \mathrm{x}(\mathrm{H}-1.3) / 3) / 10
\end{aligned}
$$

The statistical analyses were performed using SAS statistical package, Version 9.2 (SAS INSTITUTE INC., 2008). Bartlett's test was used for checking variance homogeneity (SoKAL and RoHLF, 1995), and Proc GLM for the analyses of variances according to the following statistical model:

$$
\mathrm{Y}_{i j k}=\mu+\mathrm{C}_{i}+\mathrm{B}_{j}+(\mathrm{CB})_{i j}+\varepsilon_{i j k}
$$

With:

$\mathrm{Y}_{i j k}:$ Observation on the $k^{\text {th }}$ individual of the $i^{\text {th }}$ clone
in the $j^{\text {th }}$ block; $\mu$ : Overall mean;

$\mathrm{C}_{i}$ : Effect of the factor "clone", $1 \leq i \leq 15$;

$\mathrm{B}_{j}$ : Effect of the factor "block", $1 \leq j \leq 4$;

$(\mathrm{CB})_{i j}$ : Effect of the interaction between "clone" and "block";

$\varepsilon_{i j k}$ : Residual error.

A probability level of $\mathrm{P} \leq 0.05$ was considered significant for all the statistical analyses.

Student-Newman Keuls test (SoKAL and RoHLF, 1995) was used to compare means when the effect of the factor tested was found statistically significant.

Variance components with associated standard errors SEs, and covariances were calculated from clonal plot means using SAS Proc Mixed Covtest procedure and REML method (SAS INSTITUTE INC., 2008) with block as fixed effect, whereas clone and clone $\mathrm{X}$ block were considered as random effects.

Table 2. - Descriptions of the planting site (96G, Brumas, SSSB).

\begin{tabular}{lc}
\hline Lat. & $4^{\circ} 35^{\prime} \mathrm{N}$ \\
Long. & $117^{\circ} 40^{\prime} \mathrm{W}$ \\
Elevation (meters above sea level) & $40-60 \mathrm{~m}$
\end{tabular}

Mean monthly temperatures $\quad 26-28^{\circ} \mathrm{C}$

$\begin{array}{lc}\text { Soil chemical analyses * } & \\ \text { K (me \%) } & 0.27 \\ \text { Ca (me \%) } & 1.71 \\ \mathrm{Mg}(\mathrm{me} \%) & 1.08 \\ \text { P Total (ppm) } & 206 \\ \text { P Available (ppm) } & 14.6 \\ \text { Al (me \%) } & 0.50 \\ \text { Org C (\%) } & 0.81 \\ \mathrm{~N}(\%) & 0.22 \\ \text { pH } \mathrm{H}_{2} \mathrm{O}(\text { range of variation) } & 4.5-4.9 \\ \text { CEC } & 15.9 \\ \text { Conductivity }(\mu \mathrm{s} / \mathrm{cm}) & 26.8\end{array}$

\section{Soil texture*}

$\begin{array}{lc}\text { Clay }(\%) & 34 \\ \text { Silt }(\%) & 15 \\ \text { Fine sand }(\%) & 49 \\ \text { Coarse sand }(\%) & 2 \\ & \\ & \text { Reddish-yellow } \\ & \\ & \text { Red/yellow latosols }\end{array}$

*Average values corresponding to 12 soil samples taken from 0 and $70 \mathrm{~cm}$ deep and from three different locations representing the total planted area. 
Table 3. - Mean square values, with degrees of freedom between brackets, of the model components used for assessing the influence on $\mathrm{H}, \mathrm{DBH}$ and $\mathrm{V}$ of the 15 different clones compared 1 to 7 years after planting.

\begin{tabular}{ccccc}
\hline Trait & Error & Clone (14) & Block (3) & Clone x Block (42) \\
\hline H1 & $2.33(991)$ & 25.50 & 95.15 & 20.96 \\
H2 & $2.70(958)$ & 47.14 & 83.01 & 24.83 \\
H3 & $5.03(933)$ & 90.43 & 375.32 & 57.08 \\
H4 & $4.75(883)$ & 74.27 & 858.64 & 56.52 \\
H6 & $3.48(761)$ & 91.50 & 267.18 & 42.40 \\
H7 & $3.70(758)$ & 137.61 & 532.47 & 53.41 \\
D3 & $6.82(969)$ & 175.83 & 155.26 & 111.87 \\
D4 & $9.15(972)$ & 113.93 & 1232.63 & 84.64 \\
D6 & $12.15\{761)$ & 110.53 & 291.00 & 77.33 \\
D7 & $14.35(758)$ & 136.95 & 335.72 & 95.11 \\
V3 & $350.55(906)$ & 5014.42 & 20336.88 & 2903.44 \\
V4 & $1269.78(881)$ & 14120.49 & 196292.24 & 10518.97 \\
V6 & $3937.78(761)$ & 43562.74 & 148681.57 & 31252.88 \\
V7 & $7768.61(758)$ & 86014.26 & 352000.83 & 61182.47 \\
\hline
\end{tabular}

Table 4. - Height mean values ( \pm standard error "SE", in $\mathrm{m}$ ) and coefficients of variation ("CV", in \%) of the 15 clones compared one (H1), two H2), three (H3), four (H4), six (H6) and seven (H7) years after planting. Letters distinguish means which are significantly different at $\mathrm{Po}=5 \%$.

\begin{tabular}{|c|c|c|c|c|c|c|c|c|c|c|c|c|}
\hline \multirow{2}{*}{ Clones } & \multicolumn{2}{|l|}{$\mathrm{H1}$} & \multicolumn{2}{|l|}{$\mathrm{H} 2$} & \multicolumn{2}{|l|}{$\mathrm{H3}$} & \multicolumn{2}{|l|}{$\mathrm{H} 4$} & \multicolumn{2}{|l|}{46} & \multicolumn{2}{|l|}{ H7 } \\
\hline & $\begin{array}{l}\text { Mean } \\
( \pm S E) \\
\end{array}$ & $\mathrm{CV}$ & $\begin{array}{l}\text { Mean } \\
( \pm \text { SE) }\end{array}$ & $\mathrm{CV}$ & $\begin{array}{l}\text { Mean } \\
( \pm \text { SE }) \\
\end{array}$ & $\mathrm{cV}$ & $\begin{array}{l}\text { Mean } \\
( \pm \text { SE) }\end{array}$ & $\mathrm{CV}$ & $\begin{array}{l}\text { Mean } \\
( \pm \text { SE) } \\
\end{array}$ & $\mathrm{CV}$ & $\begin{array}{c}\text { Mean } \\
( \pm S E)\end{array}$ & $\mathrm{CV}$ \\
\hline SI1 & $\begin{array}{c}4.1 \\
\{ \pm 0.3\} \text { a }\end{array}$ & 52 & $\begin{array}{c}7.0 \\
( \pm 0.3) \mathrm{a}\end{array}$ & 31 & $\begin{array}{c}11.2 \\
( \pm 0.2) \mathrm{a}\end{array}$ & 17 & $\begin{array}{c}12.9 \\
( \pm 0.4) \text { a }\end{array}$ & 28 & $\begin{array}{c}16.1 \\
( \pm 0.2) \text { a }\end{array}$ & 10 & $\begin{array}{c}19.3 \\
( \pm 0.3) \text { a }\end{array}$ & 13 \\
\hline SI2 & $\begin{array}{c}3.2 \\
( \pm 0.3) \mathrm{bcd}\end{array}$ & 67 & $\begin{array}{c}6.0 \\
( \pm 0.3) \mathrm{cd}\end{array}$ & 38 & $\begin{array}{c}7.9 \\
( \pm 0.5) \text { def }\end{array}$ & 48 & $\begin{array}{c}10.3 \\
( \pm 0.7) \mathrm{de}\end{array}$ & 45 & $\begin{array}{c}13.8 \\
( \pm 0.4) \mathrm{b}\end{array}$ & 21 & $\begin{array}{c}16.1 \\
( \pm 0.2) \mathrm{cd}\end{array}$ & 11 \\
\hline SI3 & $\begin{array}{c}3.8 \\
( \pm 0.2) \text { ab }\end{array}$ & 49 & $\begin{array}{c}6.8 \\
( \pm 0.2) \mathrm{ab}\end{array}$ & 24 & $\begin{array}{c}10.6 \\
( \pm 0.3) \mathrm{a}\end{array}$ & 21 & $\begin{array}{c}12.2 \\
( \pm 0.3) \mathrm{ab}\end{array}$ & 20 & $\begin{array}{c}15.5 \\
( \pm 0.2) \mathrm{a}\end{array}$ & 10 & $\begin{array}{c}19.0 \\
( \pm 0.4) \mathrm{a}\end{array}$ & 18 \\
\hline 514 & $\begin{array}{c}2.4 \\
( \pm 0.2) \mathrm{e}\end{array}$ & 73 & $\begin{array}{c}5.0 \\
( \pm 0.2) \text { ef }\end{array}$ & 32 & $\begin{array}{c}7.0 \\
( \pm 0.3) f\end{array}$ & 32 & $\begin{array}{c}9.7 \\
( \pm 0.4) \mathrm{e}\end{array}$ & 30 & $\begin{array}{c}11.3 \\
( \pm 0.3) \mathrm{d}\end{array}$ & 25 & $\begin{array}{c}13.6 \\
( \pm 0.3) \mathrm{e}\end{array}$ & 19 \\
\hline SI6 & $\begin{array}{c}2.3 \\
\{ \pm 0.2\} \mathrm{e}\end{array}$ & 68 & $\begin{array}{c}5.5 \\
( \pm 0.3) \mathrm{de}\end{array}$ & 39 & $\begin{array}{c}8.3 \\
( \pm 0.3) \text { bede }\end{array}$ & 25 & $\begin{array}{c}11.3 \\
( \pm 0.3) \mathrm{bcd}\end{array}$ & 21 & $\begin{array}{c}14.4 \\
( \pm 0.3) \mathrm{b}\end{array}$ & 15 & $\begin{array}{c}16.1 \\
( \pm 0.4) \mathrm{cd}\end{array}$ & 14 \\
\hline $\mathrm{SI}$ & $\begin{array}{c}2.9 \\
( \pm 0.2) \text { cde }\end{array}$ & 64 & $\begin{array}{c}5.8 \\
( \pm 0.3) \mathrm{cd}\end{array}$ & 39 & $\begin{array}{c}8.4 \\
( \pm 0.4) \text { cde }\end{array}$ & 37 & $\begin{array}{c}11.0 \\
( \pm 0.5) \mathrm{cd}\end{array}$ & 42 & $\begin{array}{c}14.3 \\
( \pm 0.3) b\end{array}$ & 18 & $\begin{array}{c}17.1 \\
( \pm 0.3) b c\end{array}$ & 15 \\
\hline Sl8 & $\begin{array}{c}4.1 \\
\{ \pm 0.3\} \text { a }\end{array}$ & 56 & $\begin{array}{c}6.6 \\
( \pm 0.3) \text { abc }\end{array}$ & 31 & $\begin{array}{c}9.8 \\
( \pm 0.6) b\end{array}$ & 44 & $\begin{array}{c}12.4 \\
( \pm 0.3) \text { a }\end{array}$ & 20 & $\begin{array}{c}13.8 \\
( \pm 0.3) \mathrm{b}\end{array}$ & 19 & $\begin{array}{c}16.1 \\
( \pm 0.3) \mathrm{cd}\end{array}$ & 13 \\
\hline MBO & $\begin{array}{c}3.4 \\
( \pm 0.2) \text { abc }\end{array}$ & 55 & $\begin{array}{c}5.9 \\
( \pm 0.2) \text { cd }\end{array}$ & 32 & $\begin{array}{c}8.9 \\
( \pm 0.3) \text { bcd }\end{array}$ & 24 & $\begin{array}{c}11.3 \\
( \pm 0.5) \text { bcd }\end{array}$ & 32 & $\begin{array}{c}14.3 \\
( \pm 0.3) b\end{array}$ & 16 & $\begin{array}{c}15.5 \\
( \pm 0.3) \mathrm{d}\end{array}$ & 14 \\
\hline DG1 & $\begin{array}{c}2.5 \\
( \pm 0.2) \mathrm{de}\end{array}$ & 56 & $\begin{array}{c}4.3 \\
( \pm 0.2) f\end{array}$ & 42 & $\begin{array}{c}7.7 \\
( \pm 0.4) \text { ef }\end{array}$ & 48 & $\begin{array}{c}11.0 \\
( \pm 0.3) \mathrm{cd}\end{array}$ & 23 & $\begin{array}{c}11.7 \\
( \pm 0.4) \mathrm{d}\end{array}$ & 21 & $\begin{array}{c}14 \\
( \pm 0.4) \mathrm{e}\end{array}$ & 18 \\
\hline DG3 & $\begin{array}{c}3.2 \\
( \pm 0.2) \mathrm{bcd}\end{array}$ & 62 & $\begin{array}{c}4.8 \\
( \pm 0.3) \text { ef }\end{array}$ & 44 & $\begin{array}{c}10.0 \\
( \pm 0.5) \text { bed }\end{array}$ & 44 & $\begin{array}{c}11.1 \\
\langle \pm 0.4) \mathrm{bcd}\end{array}$ & 32 & $\begin{array}{c}13.6 \\
( \pm 0.4) \mathrm{b}\end{array}$ & 24 & $\begin{array}{c}15.7 \\
( \pm 0.5) \mathrm{d}\end{array}$ & 23 \\
\hline MA106 & $\begin{array}{c}3.4 \\
( \pm 0.2) \text { abc }\end{array}$ & 47 & $\begin{array}{c}5.8 \\
( \pm 0.2) \mathrm{cd}\end{array}$ & 27 & $\begin{array}{c}8.7 \\
( \pm 0.3) \text { bcde }\end{array}$ & 28 & $\begin{array}{c}11.1 \\
\langle \pm 0.2) b c d\end{array}$ & 20 & $\begin{array}{c}13.8 \\
( \pm 0.3) \mathrm{b}\end{array}$ & 17 & $\begin{array}{c}15.3 \\
( \pm 0.4) \mathrm{d}\end{array}$ & 18 \\
\hline JA16 & $\begin{array}{c}3.3 \\
( \pm 0.2) b c\end{array}$ & 56 & $\begin{array}{c}6.5 \\
( \pm 0.2) \text { abc }\end{array}$ & 32 & $\begin{array}{c}9.0 \\
( \pm 0.2) \text { bcd }\end{array}$ & 20 & $\begin{array}{c}12.1 \\
( \pm 0.3) \text { abc }\end{array}$ & 24 & $\begin{array}{c}14.3 \\
( \pm 0.3) b\end{array}$ & 19 & $\begin{array}{c}17.4 \\
( \pm 0.4) b\end{array}$ & 17 \\
\hline KM29 & $\begin{array}{c}2.5 \\
( \pm 0.2) \mathrm{de}\end{array}$ & 65 & $\begin{array}{c}4.8 \\
( \pm 0.2) \text { ef }\end{array}$ & 39 & $\begin{array}{c}7.7 \\
( \pm 0.3) \text { ef }\end{array}$ & 36 & $\begin{array}{c}10.6 \\
( \pm 0.3) \mathrm{de}\end{array}$ & 27 & $\begin{array}{c}13.4 \\
( \pm 0.3) b\end{array}$ & 20 & $\begin{array}{c}16.5 \\
( \pm 0.5) \mathrm{cd}\end{array}$ & 24 \\
\hline KM41 & $\begin{array}{c}2.4 \\
( \pm 0.2) e\end{array}$ & 65 & $\begin{array}{c}4.9 \\
( \pm 0.2) \text { ef }\end{array}$ & 41 & $\begin{array}{c}7.6 \\
( \pm 0.4) \text { ef }\end{array}$ & 40 & $\begin{array}{c}10.7 \\
( \pm 0.3) \mathrm{de}\end{array}$ & 24 & $\begin{array}{c}12.6 \\
( \pm 0.4) \mathrm{c}\end{array}$ & 24 & $\begin{array}{c}16.3 \\
( \pm 0.6) \mathrm{cd}\end{array}$ & 26 \\
\hline KM44 & $\begin{array}{c}2.7 \\
( \pm 0.2) \mathrm{cde}\end{array}$ & 63 & $\begin{array}{c}6.1 \\
( \pm 0.2) \mathrm{bcd}\end{array}$ & 30 & $\begin{array}{c}9.3 \\
( \pm 0.2) b c\end{array}$ & 22 & $\begin{array}{c}12.1 \\
( \pm 0.3) \mathrm{abc}\end{array}$ & 20 & $\begin{array}{c}14.0 \\
( \pm 0.3) \mathrm{b}\end{array}$ & 16 & $\begin{array}{c}15.7 \\
( \pm 0.3) \mathrm{d}\end{array}$ & 15 \\
\hline
\end{tabular}


Broad sense individual $\mathrm{H}^{2} \mathrm{i}$ and clonal mean $\mathrm{H}^{2} \mathrm{c}$ heritabilities as well as their relevant standard errors $\mathrm{SE}_{\mathrm{H}^{2} \mathrm{i}}$ and $\mathrm{SE}_{\mathrm{H}^{2} \mathrm{c}}$ were calculated using the following equations, consistently with LOTHROP et al. (1985):

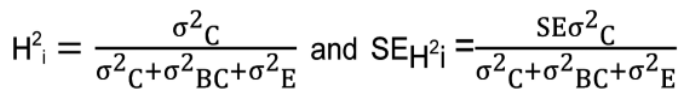

$$
\begin{aligned}
& \mathrm{H}^{2} \mathrm{c}=\frac{\sigma^{2} \mathrm{C}}{\sigma^{2} \mathrm{C}^{+\sigma^{2}} \mathrm{BC} / \mathrm{b}^{+\sigma^{2}} \mathrm{E} / \mathrm{nb}} \text { and } \mathrm{SE}_{\mathrm{H}^{2} \mathrm{c}}=\frac{\mathrm{SE} \sigma^{2} \mathrm{C}}{\sigma^{2} \mathrm{C}^{+\sigma^{2}} \mathrm{BC} / \mathrm{b}^{+\sigma^{2}} \mathrm{E} / \mathrm{nb}}
\end{aligned}
$$

With:

$\sigma_{\mathrm{C}}^{2}$ : clone genetic variance,

$\sigma^{2}{ }_{\mathrm{BC}}$ : clone (plot) $\mathrm{X}$ block interaction variance,

$\sigma_{\mathrm{E}}^{2}$ : within plot variance,

$b$ : number of blocks,

$n$ : average number of cuttings per plot,

$\mathrm{SE} \sigma_{\mathrm{C}}^{2}$ : clone genetic variance standard error.

The selection differential $\mathrm{S}$ and the predicted genetic gain $\Delta \mathrm{G}$ were calculated for the three best clones selected out of the 15 assessed (top $20 \%$ of the population), applying the following formula (ZOBEL and TALBERT, 1984; WHITE et al., 2007):

$$
\Delta \mathrm{G}=H_{C}^{2} \mathrm{~S},
$$

SAS Proc Corr procedure (SAS INSTITUTE INC., 2008) was used for assessing the phenotypic correlations $r_{\mathrm{P}}$, while the genetic correlations $r_{G}$ and their standard error $\mathrm{SEr}_{\mathrm{G}}$ were drawn from the following equations (FAlCONER, 1974; ZoBEL and TALBERT, 1984):

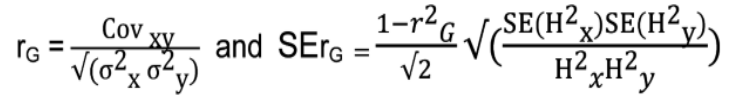

With

$\operatorname{Cov}_{\mathrm{xy}}$ : Clone covariance component for traits $\mathrm{x}$ and $\mathrm{y}$,

$\sigma_{\mathrm{x}}^{2}$ : Clone variance component for trait $\mathrm{x}$,

$\sigma_{\mathrm{y}}^{2}:$ Clone variance component for trait $\mathrm{y}$,

$\mathrm{SE}\left(\mathrm{H}^{2} \mathrm{x}\right)$ : standard error of broad sense heritability $\left(\mathrm{SE}_{\mathrm{H}_{2} \mathrm{i}}\right)$ for trait $\mathrm{x}$,

$\mathrm{SE}\left(\mathrm{H}^{2} \mathrm{x}\right)$ : standard error of broad sense heritability $\left(\mathrm{SE}_{\mathrm{H}_{2} \mathrm{i}}\right)$ for trait $\mathrm{y}$.

\section{Results}

Mortality occurred mainly during the first months following the planting and was less than $10 \%$ on average. It did not vary significantly between clones, contrary to height, $\mathrm{DBH}$ and volume for which strong clonal influences $(P<0.0001)$, block effects $(P<0.0001)$ and block $X$

Table 5. - Diameter at breast height, or "DBH", mean values ( \pm standard error "SE", in $\mathrm{cm}$ ) and coefficients of

\begin{tabular}{|c|c|c|c|c|c|c|c|c|}
\hline \multirow{2}{*}{ Clones } & \multicolumn{2}{|l|}{ D3 } & \multicolumn{2}{|l|}{ D4 } & \multicolumn{2}{|l|}{ D6 } & \multicolumn{2}{|l|}{ D7 } \\
\hline & Mean ( \pm SE) & $\mathrm{cV}$ & Mean ( \pm SE) & cV & Mean ( \pm SE) & $\mathrm{cV}$ & Mean ( \pm SE) & $\mathrm{cV}$ \\
\hline SI1 & $10.7( \pm 0.3) \mathrm{a}$ & 22 & $13.9( \pm 0.3) \mathrm{bcd}$ & 22 & $19.1( \pm 0.4) \mathrm{ab}$ & 15 & $21.3( \pm 0.4) \mathrm{bcd}$ & 14 \\
\hline $\mathrm{SI} 2$ & $8.1( \pm 0.4)$ de & 41 & $11.7( \pm 0.4) \mathrm{gh}$ & 29 & $17.2( \pm 0.5) b c$ & 21 & $19.6( \pm 0.5) \mathrm{d}$ & 20 \\
\hline $\mathrm{S} 13$ & $11.1( \pm 0.3) \mathrm{a}$ & 23 & $15.8( \pm 0.4) \mathrm{a}$ & 19 & $20.0( \pm 0.4) \mathrm{a}$ & 18 & $22.5( \pm 0.4) a b$ & 17 \\
\hline S14 & $7.8( \pm 0.3) \mathrm{de}$ & 39 & $12.4( \pm 0.5) \operatorname{defg}$ & 35 & $17.3( \pm 0.5) b c$ & 24 & $19.6( \pm 0.5) \mathrm{d}$ & 22 \\
\hline SI6 & $8.4( \pm 0.4)$ cde & 36 & $13.6( \pm 0.5)$ bcode & 29 & $18.9( \pm 0.6) \mathrm{ab}$ & 22 & $22.3( \pm 0.8)$ abc & 22 \\
\hline S17 & $8.4( \pm 0.3) \mathrm{cde}$ & 35 & $12.9( \pm 0.4) \mathrm{cdefg}$ & 28 & $17.8( \pm 0.4) b c$ & 20 & $20.3( \pm 0.5)$ bcd & 19 \\
\hline S18 & $10.2( \pm 0.4) a b$ & 36 & $14.8( \pm 0.6) \mathrm{ab}$ & 31 & $19.1( \pm 0.6) a b$ & 23 & $21.5( \pm 0.5)$ bcd & 21 \\
\hline MBO & $9.5( \pm 0.4) b c$ & 33 & $14.4( \pm 0.5) b c$ & 26 & $20.2( \pm 0.7) \mathrm{a}$ & 21 & $23.4( \pm 0.7) a$ & 20 \\
\hline DG1 & $7.1( \pm 0.3) \mathrm{e}$ & 37 & $10.8( \pm 0.3) \mathrm{h}$ & 27 & $14.5( \pm 0.6) \mathrm{d}$ & 27 & $16.3( \pm 0.6) \mathrm{e}$ & 26 \\
\hline DG3 & $9.3( \pm 0.5)$ bc & 42 & $13.4( \pm 0.6)$ cde & 37 & $17.8( \pm 0.7) b c$ & 29 & $20.1( \pm 0.8) \mathrm{cd}$ & 29 \\
\hline MA106 & $8.7( \pm 0.4) \mathrm{cd}$ & 34 & $12.6( \pm 0.5)$ defg & 32 & $18.9( \pm 0.5) \mathrm{ab}$ & 19 & $21.0( \pm 0.6)$ bcd & 20 \\
\hline JA16 & $8.0( \pm 0.6) \mathrm{de}$ & 39 & $12.3( \pm 0.4) \mathrm{efg}$ & 28 & $16.2( \pm 0.5) \mathrm{c}$ & 23 & $19.2( \pm 0.5) \mathrm{d}$ & 22 \\
\hline KM29 & $7.9( \pm 0.4)$ de & 49 & $11.8( \pm 0.6) \mathrm{fgh}$ & 44 & $18.3( \pm 0.5) \mathrm{ab}$ & 22 & $21.2( \pm 0.6) \mathrm{bcd}$ & 21 \\
\hline KM41 & $6.0( \pm 0.6) f$ & 78 & $13.3( \pm 0.6)$ cdef & 38 & $17.5( \pm 0.9) b c$ & 34 & $20.6( \pm 0.9) \mathrm{bcd}$ & 30 \\
\hline KM44 & $4.6( \pm 0.6) \mathrm{g}$ & 107 & $12.8( \pm 0.4)$ defg & 26 & $17.7( \pm 0.6) b c$ & 21 & $20.6( \pm 0.7) \mathrm{bcd}$ & 22 \\
\hline
\end{tabular}
variation ("CV", in \%) of the 15 clones compared three (D3), four (D4), six (D6) and seven (D7) years after planting. Letters distinguish means which are significantly different at $\mathrm{Po}=5 \%$. 
Table 6. - Volume mean values ( \pm standard error "SE", in $\mathrm{dm}^{3}$ ) and coefficients of variation ("CV", in \%) of the 15 clones compared three (V3), four (V4), six (V6) and seven (V7) years after planting. Letters distinguish means which are significantly different at $\mathrm{Po}=5 \%$.

\begin{tabular}{|c|c|c|c|c|c|c|c|c|}
\hline \multirow{2}{*}{ Clones } & \multicolumn{2}{|l|}{ V3 } & \multicolumn{2}{|l|}{ v4 } & \multicolumn{2}{|l|}{ v6 } & \multicolumn{2}{|l|}{ v7 } \\
\hline & Mean ( \pm SE) & $\mathrm{CV}$ & Mean $( \pm$ SE) & $\mathrm{CV}$ & Mean ( \pm SE) & $\mathrm{cV}$ & Mean ( \pm SE) & $\mathrm{cV}$ \\
\hline SI1 & $44.1( \pm 2.6) \mathrm{ab}$ & 50 & $85.9( \pm 5.4) b c$ & 51 & $183.6( \pm 7.6) \mathrm{abc}$ & 32 & $263.8( \pm 9.5)$ abc & 28 \\
\hline $\mathrm{sl2}$ & $26.4( \pm 2.7) \mathrm{def}$ & 81 & $60.0( \pm 7.5) \mathrm{de}$ & 85 & $139.3( \pm 8.9)$ def & 46 & $198.4( \pm 11.4)$ de & 42 \\
\hline $\mathrm{sl} 3$ & $45.9( \pm 2.7) a$ & 50 & $101.4( \pm 5.9) \mathrm{a}$ & 44 & $197.6( \pm 9.5) \mathrm{a}$ & 39 & $298.0( \pm 14.2) \mathrm{a}$ & 39 \\
\hline SI4 & $19.9( \pm 2.0)$ ef & 82 & $56.0( \pm 5.4) \mathrm{de}$ & 75 & $122.5( \pm 9.0) \mathrm{f}$ & 58 & $179.0( \pm 11.1) \mathrm{e}$ & 49 \\
\hline \$16 & $25.7( \pm 2.5) \mathrm{def}$ & 76 & $75.1( \pm 5.8) \mathrm{cd}$ & 60 & $170.1( \pm 11.8)$ abcd & 44 & $262.6( \pm 18.3)$ abc & 44 \\
\hline SI7 & $26.5( \pm 2.4) \mathrm{def}$ & 77 & $72.0( \pm 5.6) \mathrm{cd}$ & 65 & $150.4( \pm 8.5)$ cdef & 45 & $225.0( \pm 12.4)$ bcde & 44 \\
\hline S18 & $40.7( \pm 4.3)$ ab & 82 & $95.6( \pm 7.4)$ ab & 62 & $174.6( \pm 11.1)$ abcd & 51 & $240.8( \pm 12.6)$ bcd & 42 \\
\hline MBO & $31.3( \pm .2 .8) \mathrm{cd}$ & 73 & $82.6( \pm 6.1) b c$ & 57 & $192.2( \pm 13.2) a b$ & 43 & $273.0( \pm 17.6) \mathrm{ab}$ & 41 \\
\hline DG1 & $17.7( \pm 1.8) f$ & 86 & $47.3( \pm 3.6) \mathrm{e}$ & 64 & $89.0( \pm 7.4) \mathrm{g}$ & 57 & $128.9( \pm 10.9) \mathrm{f}$ & 58 \\
\hline DG3 & $36.8( \pm 4.3) \mathrm{bc}$ & 84 & $82.7( \pm 7.7)$ bc & 73 & $156.7( \pm 13.2)$ cdef & 63 & $221.4( \pm 17.5)$ bcde & 59 \\
\hline MA106 & $26.0( \pm 2.3)$ def & 69 & $66.8( \pm 5.3) \mathrm{cd}$ & 63 & $162.5( \pm 9.4)$ bcde & 43 & $223.6( \pm 13.7)$ bcde & 45 \\
\hline JA16 & $24.1( \pm 2.4)$ def & 86 & $62.6( \pm 4.5)$ bcd & 60 & $127.5( \pm 9.1)$ ef & 57 & $208.9( \pm 14.4) \mathrm{de}$ & 55 \\
\hline KM29 & $22.8( \pm 3.7) \mathrm{def}$ & 131 & $66.6( \pm 8.0) \mathrm{cd}$ & 100 & $153.9( \pm 10.8) \mathrm{cdef}$ & 55 & $244.9( \pm 17.1)$ bcd & 54 \\
\hline KM41 & $27.1( \pm 3.1) \mathrm{def}$ & 89 & $72.1( \pm 6.3) \mathrm{cd}$ & 69 & $147.5( \pm 13.9) \mathrm{cdef}$ & 64 & $241.5( \pm 21.7)$ bcd & 60 \\
\hline KM44 & $28.5( \pm 2.1) \mathrm{de}$ & 60 & $70.3( \pm 5.1) \mathrm{cd}$ & 60 & $146.2( \pm 11.8) \mathrm{cdef}$ & 53 & $218.6( \pm 18.4) \mathrm{cde}$ & 55 \\
\hline
\end{tabular}

clone interactions $(\mathrm{P}<0.0001)$ were noticeable from one to seven years after planting (see Table 3 for relevant mean squares). At each recording date, SI1 was the taller of the 15 clones compared, followed by SI3, reaching at age 7 average heights of $19.3 \mathrm{~m}$ and $19 \mathrm{~m}$ respectively (Table 4). This corresponded to mean annual increment in height (MAIH) of more than $2.7 \mathrm{~m}$, whereas lower values were recorded for clones SI4 (13.6 $\mathrm{m}$ and MAIH of $1.9 \mathrm{~m}$ ) and DG1 (14 m and MAIH of $2 \mathrm{~m}$ ). The higher $\mathrm{DBH}$ values 3 and 4 years after planting were 11.1 and $15.8 \mathrm{~cm}$ for SI3, 10.2 and $14.8 \mathrm{~cm}$ for SI 8 and 10.7 and $13.9 \mathrm{~cm}$ for SI1, respectively, supplanted by MBO at age $6(20.2 \mathrm{~cm})$ and age $7(23.4 \mathrm{~cm})($ Table 5$)$. The lower DBH scores recorded after 3 years for clones $\mathrm{KM} 44(4.6 \mathrm{~cm})$ and $\mathrm{KM} 41(6.0 \mathrm{~cm})$ increased markedly with age, whereas DG1 DBH remained low from 3 to 7 years after planting. Mean annual increment in $\mathrm{DBH}$ (MAIDBH) 7 years after planting ranged from $2.3 \mathrm{~cm}$ for DG1 to $3.3 \mathrm{~cm}$ for MBO. Consistently with these height and $\mathrm{DBH}$ scores, the higher volume values recorded 7 years after planting were $298 \mathrm{dm}^{3}$ for clone SI3, $273 \mathrm{dm}^{3}$ for clone $\mathrm{MBO}$, and $263.8 \mathrm{dm}^{3}$ for clone SI1 whereas clones DG1, SI4 and SI2 scored lower values of 128.9,
179 and $198.4 \mathrm{dm}^{3}$ respectively (Table 6). This corresponded to varying mean annual increment in volume (MAIV) from $18.4 \mathrm{dm}^{3}$ for clone DG1 to $42.6 \mathrm{dm}^{3}$ for clone SI3 after 7 years.

Overall, for all three growth traits, broad sense heritability estimates were associated with high standard errors and were noticeably lower for single trees $\mathrm{H}^{2} \mathrm{i}$ than for clonal means $\mathrm{H}^{2} \mathrm{c}\left(\mathrm{H}^{2} \mathrm{i} \leq 0.257 \mathrm{vs} \mathrm{H^{2 }} \mathrm{c} \leq 0.634\right.$ for height, $\mathrm{H}^{2} \mathrm{i} \leq 0.120$ vs $\mathrm{H}^{2} \mathrm{c} \leq 0.383$ for $\mathrm{DBH}$ and $\mathrm{H}^{2} \mathrm{i} \leq$ 0.125 vs $\mathrm{H}^{2} \mathrm{c} \leq 0.364$ for volume, Table 7$) . \mathrm{H}^{2} \mathrm{i}$ and $\mathrm{H}^{2} \mathrm{c}$ estimates increased as trees reached 6 and 7 years for height only.

The highest genetic gain that could be expected based on the selection of the three best clones was at age 2 for height $(+0.66 \mathrm{~m}$, or $+11.7 \%)$, at age 3 for $\mathrm{DBH}$ $(+0.87 \mathrm{~cm}$, or $+10.4 \%)$ and volume $\left(+4.65 \mathrm{dm}^{3}\right.$, or $+15.7 \%$ ) (Table 7 ).

Age-related phenotypic correlation values were reliably $(\mathrm{P}<0.0001)$ higher and more consistent for $\mathrm{DBH}$ $\left(r_{\mathrm{P}} \geq 0.61\right)$ than for height $\left(0.37 \leq \mathrm{r}_{\mathrm{P}} \leq 0.69\right)$, or than between $\mathrm{DBH}$ and height except for $\mathrm{H} 3\left(0.51 \leq \mathrm{r}_{\mathrm{P}} \leq 0.63\right)$ and for $\mathrm{H} 6\left(0.55 \leq \mathrm{r}_{\mathrm{P}} \leq 0.69\right)$ (Table 8). Genetic correla- 
Table 7. - Broad sense individual $\mathrm{H}^{2} \mathrm{i}$, and clonal mean $\mathrm{H}^{2} \mathrm{c}$ heritability estimates ( \pm standard error "SE") for the different traits assessed, and relevant estimated genetic gain $\Delta \mathrm{G}$ (actual values and \% to the mean value) calculated for the 3 best clones out of the 15 recorded.

\begin{tabular}{|c|c|c|c|}
\hline Trait & $\mathrm{H}^{2} \mathbf{i}( \pm \mathrm{SE})$ & $H^{2} \mathrm{c}( \pm \mathrm{SE})$ & $\Delta G(\%)$ \\
\hline $\mathrm{H} 1$ & $0.062( \pm 0.126)$ & $0.318( \pm 0.651)$ & $0.28 \mathrm{~m}(9.2 \%)$ \\
\hline $\mathrm{H} 2$ & $0.182( \pm 0.155)$ & $0.606( \pm 0.514)$ & $0.66 \mathrm{~m}(11.7 \%)$ \\
\hline H3 & $0.111( \pm 0.136)$ & $0.386( \pm 0.472)$ & $0.69 \mathrm{~m}(7.9 \%)$ \\
\hline $\mathrm{H} 4$ & $0.095( \pm 0.131)$ & $0.344( \pm 0.473)$ & $0.41 \mathrm{~m}(3.6 \%)$ \\
\hline $\mathrm{H} 6$ & $0.200( \pm 0.156)$ & $0.564( \pm 0.440)$ & $0.85 \mathrm{~m}(6.2 \%)$ \\
\hline $\mathrm{H} 7$ & $0.257( \pm 0.172)$ & $0.634( \pm 0.422)$ & $1.47 \mathrm{~m}(9.1 \%)$ \\
\hline D3 & $0.120( \pm 0.137)$ & $0.383( \pm 0.436)$ & $0.87 \mathrm{~cm}(10.4 \%)$ \\
\hline D4 & $0.082( \pm 0.128)$ & $0.297( \pm 0.465)$ & $0.57 \mathrm{~cm}(4.3 \%)$ \\
\hline D6 & $0.097( \pm 0.132)$ & $0.331( \pm 0.449)$ & $0.57 \mathrm{~cm}(3.1 \%)$ \\
\hline D7 & $0.101( \pm 0.133)$ & $0.336( \pm 0.438)$ & $0.71 \mathrm{~cm}(3.4 \%)$ \\
\hline V3 & $0.110( \pm 0.135)$ & $0.332( \pm 0.407)$ & $4.65 \mathrm{dm}^{3}(15.7 \%)$ \\
\hline v4 & $0.125( \pm 0.137)$ & $0.364( \pm 0.400)$ & $7.71 \mathrm{dm}^{3}(9.6 \%)$ \\
\hline v6 & $0.099( \pm 0.132)$ & $0.306( \pm 0.407)$ & $11.28 \mathrm{dm}^{3}(7.3 \%)$ \\
\hline$v 7$ & $0.096( \pm 0.131)$ & $0.274( \pm 0.376)$ & $13.62 \mathrm{dm}^{3}(5.9 \%)$ \\
\hline
\end{tabular}

Table 8. - Genetic (above diagonal, with standard error between brackets) and phenotypic (below diagonal, $\mathrm{P}<0.0001$ ) correlation coefficient estimates for the different traits assessed.

\begin{tabular}{|c|c|c|c|c|c|c|c|c|c|c|}
\hline Trait & H1 & H2 & H3 & H4 & H6 & H7 & D3 & D4 & D6 & D7 \\
\hline H1 & & $\mathbf{0 . 9 0 ( 0 . 0 3 )}$ & $1.30(-0.79)$ & $1.19(-0.49)$ & $\mathbf{0 . 8 0 ( 0 . 2 0 )}$ & $0.68(0.45)$ & $1.37(-0.95)$ & $0.37(1.11)$ & $0.00(1.11)$ & $-0.54(0.82)$ \\
\hline H2 & 0.57 & & $0.92(\mathbf{0 . 1 1})$ & $1.13(-0.21)$ & $1.14(-0.74)$ & $0.94(0.06)$ & $\mathbf{0 . 5 4 ( 0 . 4 9 )}$ & $\mathbf{0 . 6 3 ( 0 . 5 0 )}$ & $\mathbf{0 . 5 5 ( 0 . 5 2 )}$ & $0.41(0.99)$ \\
\hline H3 & 0.47 & 0.52 & & $2.17(-3.4)$ & $\mathbf{0 . 9 6 ( 0 . 0 5 )}$ & $0.89(0.13)$ & $\mathbf{0 . 5 9 ( 0 . 5 4 )}$ & $0.59(0.63)$ & $-0.07(0.90)$ & $0.34(0.79)$ \\
\hline H4 & 0.37 & 0.45 & 0.59 & & $1.22(-0.36)$ & $1.17(-0.25)$ & $\mathbf{0 . 9 0}(0.16)$ & $1.48(-1.23)$ & $0.44(0.78)$ & $0.32(0.85)$ \\
\hline H6 & 0.41 & 0.50 & 0.63 & 0.54 & & $0.95(0.05)$ & $0.46(0.53)$ & $\mathbf{0 . 6 1 ( 0 . 4 9 )}$ & $0.36(0.64)$ & $0.37(0.62)$ \\
\hline H7 & 0.37 & 0.41 & 0.51 & 0.39 & 0.69 & & $\mathbf{0 . 5 8 ( 0 . 4 1 )}$ & $0.44(0.58)$ & $0.11(0.66)$ & $0.12(0.66)$ \\
\hline D3 & 0.50 & 0.48 & 0.56 & 0.31 & 0.55 & 0.46 & & $\mathbf{0 . 7 2 ( 0 . 4 5 )}$ & $0.57(0.60)$ & $0.09(0.86)$ \\
\hline D4 & 0.55 & 0.50 & 0.60 & 0.49 & 0.59 & 0.52 & 0.68 & & $\mathbf{0 . 9 4 ( 0 . 1 2 )}$ & $\mathbf{0 . 8 6 ( 0 . 2 6 )}$ \\
\hline D6 & 0.48 & 0.48 & 0.60 & 0.41 & 0.66 & 0.49 & 0.66 & 0.76 & & $\mathbf{0 . 9 3 ( 0 . 1 2 )}$ \\
\hline D7 & 0.43 & 0.45 & 0.56 & 0.38 & 0.64 & 0.51 & 0.61 & 0.73 & 0.94 & \\
\hline
\end{tabular}

Genetic correlation coefficient estimates in bold only seem reliable, contrary to the others in italic with high standard error, too uncertain when not erroneous ( $>1)$.

tion estimates were overall higher than phenotypic correlation coefficients, and indicated stronger positive relationships between materials of different ages for height than for DBH. This is particularly true for the genetic correlation estimates associated to low standard errors, hence more reliable, which ranged between 0.80 and 0.96 , whereas height and $\mathrm{DBH}$ appeared to be moderately to highly genetically correlated $\left(0.54 \leq \mathrm{r}_{\mathrm{G}} \leq 0.90\right)$.

\section{Discussion}

\section{Growth differences}

Despite a striking development of teak clonal plantations from rooted cuttings or microcuttings during the past few years (SMIT et al., 2011; GoH and MonTEUUIS, 2012), growth assessment of teak clones produced by these vegetative propagation methods remains scarce 
and fragmentary (SiSWAMARTANA and Wibowo, 2005; CALlister and Collins, 2008; WARDANI et al., 2009). It was limited to budded clones (SUKSILEUNG et al., 1975; HARSHAP and SOERIANEGARA, 1977) when grafting was the only practical way of producing teak clones from mature selected genotypes for the main purpose of establishing clonal seed orchards (MONTEUUIS and GoH, 1999). To our knowledge, comparative growth performances arising from 7 years of observations under humid field conditions of teak clones produced by micropropagation from 6 month- to more than 60 year-old ortets has never been published before. This study shows first that teak plants produced from microcuttings further to the formation of an adventitious root system can be field planted with similar, if not higher, success rates than tap-rooted seedlings (CHAIX et al., 2011; MonTEUUIS et al., 2011). Such good survival could be due to the high rainfalls to which the planting material was exposed in the absence of a dry distinct season, in contrast to other sites where post-planting mortality rates were higher (MADOFFE and MAGHEMBE, 1988; KAOSA-ARD, 2000; BEKKER et al., 2004). Similar to seedderived populations (MoNTEUUIS et al. 2011), these abundant precipitations could have also accounted for the unusual growth rates recorded, which could have been even higher in more suitable soil conditions (CHAIX et al., 2011). In comparison, in northern Thailand, under precipitations of 1000 to $1100 \mathrm{~mm} / \mathrm{yr}$ with a 4 monthlong dry season, SUKSILEUNG et al. (1975) reported heights of 7.7 to $10.7 \mathrm{~m}$ after 7 years for phenotypicallysuperior clones established as clonal seed orchards in two different sites, but these clones were grafted (budded). Our clones were twice as tall at the same age. In Kerala, India, also under mean annual rainfall of $2500 \mathrm{~mm}$, the best MAIH was $2.4 \mathrm{~m}$ after 3 years for a rooted-cutting clone derived from a locally selected mature ortet (PALANISAMY et al., 2009). In Java, Indonesia, NA'IEM (2001) reported heights ranging from 2.7 to $3.5 \mathrm{~m}$ after 16 months for 20 clones produced by rooted cuttings from mature selected ortets and established in four different sites with rainfalls of 1700 to $2900 \mathrm{~mm} / \mathrm{yr}$. In the same sites and for the same kind of clonal material but 5 year after planting, mean height varied from 6.1 to $8.2 \mathrm{~m}$, and DBH from $6.2 \mathrm{~cm}$ to $10.1 \mathrm{~cm}$, with the best performances for the wetter conditions (SISWAMARTANA and WiBOwo, 2005). These scores, even under higher rainfall regimes, remained lower than our records, with MAIH of more than $3.5 \mathrm{~m}$ after 3 years, and of more than $2.5 \mathrm{~m}$ after 7 years for clones SI1 and SI3. These Solomon Island-derived clones seemed to thrive surprisingly under humid conditions, and also under a much wider range of planting conditions, including sites exposed to more constraining rainfall regimes elsewhere (GOH and MonteuUis, 2012). Deployed in mixture or "in bulk", they have outperformed so far all the other teak origins to which they had been compared in the same field conditions (GoH and MonTEUUIS, 2012). These clones originated from a single bunch of a few fruits and are assumed to be genetically closely related based on this observation and DNA investigations ( $\mathrm{GoH}$ et al., 2007). In spite of this, this study shows that they could differ significantly in height, DBH and volume. Such noteworthy variations were already reported among half-sibs for height but also for other phenotypic and wood traits in teak. This pleads for the use of judiciously selected clones rather than of seedlings for establishing superior yield and quality plantations for this species (MonteuUIS and GoH, 1999; GoH and MonteuUIS, 2005; GoH et al., 2007). This view is supported by the possibility to clone true-to-type teak selected ortets of varying ages in absence of C-effects (LIBBY and JUND, 1962; TIMMIS et al., 1987) and of negative ageing influence on growth rate (BONGA, 1982; FRAMPTON and FosTER, 1993; MoNTEUUIS and GoH, 1999). These factors could partly explain the poor growth performances and great withinclone variability noticed by SUKSILEUNG et al. (1975) for budded clones. Grafting is classically used for clonally propagating genotypes which are physiologically too mature to form adventitious roots and hence to be multiplied by rooted cuttings (MONTEUUIS, 1985; HARTMANN et al., 1997). In addition to maturation-induced negative effects on shoot development, like topophysis (OLESEN 1978), differences in root-stock vigor, in genotypic compatibility with the grafted scion, and also in the quality of the graft union are prone to amplify C effects (BuRDON and SHELBOURNE, 1974; LIBBy, 1974). The fact that clones produced from 27 to 67 yr-old ortets displayed higher growth capacities than DG1 and DG3 clones derived from 6 month-old seedlings is a striking demonstration that mature selected teak genotypes can be physiologically rejuvenated providing suitable propagation methods are used. These methods were more efficient and conservative than felling the selected ortets with the expectations to stimulate the production of stump sprouts that can be used as cuttings (PALANISAMY et al., 2009). This also strengthens the benefits of developing teak clones from mature Plus trees instead of from seedlings, much too young to express traits of economical importance that can be profitably used for selection (BONGA, 1982; ZoBEL and TALBERT, 1984; Timmis et al., 1987). Certain of these traits like stem form, bole shape, buttressing or fluting, and wood characteristics obviously require longer growth period than seven years to be reliably assessed (KJAER and LAURIDSEN, 1996). This is why we decided to restrict our clonal test assessment to three growth criteria of great economical impact: height, $\mathrm{DBH}$ and volume, less subjective to assess than more qualitative traits (ZOBEL and TALBERT, 1984; WHITE et al., 2007), notwithstanding the very good stem form of most of the clones observed. Similar coefficients of variation of young (DG1, DG3) and mature (MBO, KM29) genotypes for these traits is another indication that old teak trees can be cloned by appropriate vegetative methods in the absence of maturationinduced $\mathrm{C}$ effects. These are responsible for the detrimental intraclonal variability commonly associated with the cloning of most arborescent species (LIBBY and JUNG, 1962; BONGA, 1982; WHITE et al., 2007).

\section{Genetic parameter estimates}

Genetic parameters are theoretically more accurately assessed from clones than from family-derived populations, as every clonal offpring has rigorously the same genetic make-up as the original ortet from which they initially derive comprising of the entire genetic variance 
through its additive and non-additive components plus gene interactions (LIBBY and JUNG, 1962). This is a big difference with seedlings irrespective of their genetic relatedness (Burdon and Shelbourne, 1974; FrAmpton and Foster, 1993). Further to previous assumptions (GOGATE et al., 1997), the importance of non-additive effects in the genetic control of growth traits in teak was recently confirmed by CALLISTER and CoLLINS (2008).

The much lower values obtained for broad sense heritabilities assessed from single trees $\mathrm{H}^{2} \mathrm{i}$ versus from clonal mean $\mathrm{H}^{2}$ c, as observed by WARDANI et al. (2009) in Java, illustrate that the evaluation based on non-replicated individuals is likely to be biased by the microenvironment. This emphasizes the importance of increasing the number of clonal replicates, especially on heterogeneous sites, for more accurate estimates then higher genetic improvement efficiency (FRAMPTON and FOSTER, 1993). But the prime objective of this study was to compare on a rough piece of land the growth performances of a limited number of clones derived from mature selected ortets. This is why a layout consisting of clonal plots large enough for reducing the competition with genetically different surrounding trees while allowing a better assessment of within-clone variation was adopted (LIBBY and JUND, 1962). Experimental designs with a greater number of clones, not necessarily deriving from selected from Plus trees, but each represented by a few number of ramets (lower within-plot environmental variation) per multiple replicate planted in more homogenous blocks would have very likely resulted in more accurate evaluations (FRAMPTON and FOSTER, 1993; GEZAN et al., 2006; SolÓRZANO NARANJO et al., 2012). For instance, CALLISTER and CoLLINS (2008) used for assessing teak genetic parameters a clonally replicated progeny test encompassing 696 different clones, each represented by replicates of 1 to 4 ramets planted within a complete random block design. In teak as in many other tree species (WHITE et al., 2007), height, DBH and volume heritability estimates were observed to vary markedly from one site to another (SISWAMARTANA and WIBOWO, 2005; WARDANI et al., 2009), as well as according to age in similar environment for clonal seed orchard families (CHAIX et al., 2011; MonteUUIS et al., 2011). Such age-related variations could be induced by changes for light competition between nearby trees, amplified by the uneven topography of the planting site. Notwithstanding high standard errors, our $\mathrm{H}^{2} \mathrm{c}$ estimates are consistent for $\mathrm{DBH}$ with the values obtained on the same species by SOLÓRZANO NARANJO et al. (2012) from a 4 yr-old replicated clonal test, and slightly higher for height than the values reported by CALLISTER and Collins (2008) for 3.5 yr-old teak clones. The higher estimates reported by SISWAMARTANA and WiBOwO (2005), then by WARDANI et al. (2009) might be due, at least partly, to the plagiotropic C-effects noticed by these authors and which are liable to bias upwardly heritabilities (LIBBY and JUND, 1962; FRAMPTON and FosTER, 1993; WHITE et al., 2007). Grafting-amplified C-effects could also explain the surprisingly high broad sense heritability values obtained for height (0.67) and DBH (0.87) by HARSHAP and SOERIANEGARA (1977) from 25-yr old budded clones.
Aside from heritability magnitude, increasing the selection differential value would have been another means of enhancing the genetic gain. In this respect, higher efficiency could have been obtained using as base population a greater number of unselected genotypes from which a smaller proportion of top ones could be selected with a higher intensity as subpopulation (BAGCHI, 1995; WARDANI et al., 2009). Assessing genetic gains from selected clones produced true-to-type by rooted microcuttings from mature ortets is more unusual in forest genetics (ZOBEL and TALBERT, 1984; FRAMPTON and Foster, 1993; White et al., 2007).

Site heterogeneity and experimental design flaws are very likely also responsible for the moderate phenotypic correlation values found for height and $\mathrm{DBH}$, compared to other studies benefitting from more adapted layout and even environmental conditions ((CALLISTER and Collins, 2008). In the rough site conditions of Luasong (MoNTEUUIS et al., 2011), the phenotypic correlation estimates obtained for seed-derived populations were also overall lower than in Taliwas which is on a more uniform and flatter topography (CHAIX et al., 2011). The higher phenotypic correlation values observed between height and $\mathrm{DBH}$ for $\mathrm{H} 3$ and $\mathrm{H} 6$ could account for the similarities observed in clone ranking at ages 3 and 6 for height, $\mathrm{DBH}$ and volume. Overall, estimated values were lower for the phenotypic than for the genetic correlations as already observed in teak (SUKSILEUNG et al., 1975; WARDANI et al., 2009), and more generally, for various plant and animal species (SEARLE, 1961; Cheverud, 1988). Genetic correlations are very difficult to assess reliably for forest tree species (WHITE et al., 2007), and as such, unexpectedly low or high (> 1) estimates associated to high or negative standard errors values, which cannot exist by definition, were ignored. This problem, reported in the literature for teak (SUKSILEUnG et al., 1975; KJAER et al., 1995; KJAER and LAURIDSEN, 1996) and for quite different species (АввотT and SvEnsSON, 2010), is very likely due to experimental design defects resulting in odd covariance values that were used for calculating the genetic correlation estimates. More clones scattered in a greater number of site replicates or plots would have probably here again resulted in more accurate and reliable evaluations (FALCONER and MACKEY, 1996; White et al., 2007). The moderate to strong positive estimates found among growth traits, although logically expected (WHITE et al., 2007), were also observed between height and $\mathrm{DBH}$ for 4 yr-old teak clones (SolóRZANO NARANJO et al., 2012). Extension of genetic correlation analyses to other traits, like stem form and age to age evaluations (KJAER et al., 1995; KJAER and LAURIDSEN, 1996) appears highly advisable for knowing if and how soon these characters of great economical impact of teak can be reliably predicted from growth data. As far as we are aware, the only relevant information available to date is that genetic correlations between growth and stem form were 0.73 for 12 yr-old seed-derived teak trees (DANARTO and HARDIYANTO, 2001), whereas CALlister and Collins (2008) indicated correlations between volume and straightness were 0.40 for seedlings and 0.32 for clones 3.5 years after planting. 


\section{Conclusion}

This study provides original information on growth performances of teak clones produced by microcuttings from young and mature selected ortets and planted under wet tropical conditions. It indicates also to what extent growth parameters in teak can be assumed to be genetically controlled, strengthening and enriching thereby previous findings. This completes recent observations on the behaviour of various teak seed populations established in similar environment (CHAIX et al., 2011; MonteuUis et al., 2011). Lastly, this work demonstrates for the first time the practical efficiency of suitable micropropagation methods for cloning phenotypically superior teak trees of various ages, anticipating bright prospects for teak clonal forestry under high rainfall regimes.

\section{Acknowledgements}

The authors are very grateful to Dr. GARTH NIKLES for his valuable comments and suggestions on an early draft of this paper.

\section{References}

Аввотт, J. K. and E. I. Svensson (2010): Morph-specific variation in intersexual genetic correlations in an intraspecific mimicry system. Evolutionary Ecology Research 12: 105-118.

BAGCHI, S. K. (1995): Selection differential and predicted genetic gain in Tectona grandis. The Indian Forester 121(6): 482-490.

Ball, J. B., D. PANDey and S. Hirai (2000): Global overview of teak plantations. In: „Site, technology and productivity of teak plantations“. FORSPA Publication $\mathrm{N}^{\circ}$ 24/2000, TEAKNET Publication $\mathrm{N}^{\circ} 3,11-33$.

BekKer, C., W. RANCE and O. MonteuUis (2004): Teak in Tanzania: the Kilombero Valley Teak Co. Ltd. Project. Bois et Forêts des Tropiques 279: 11-21.

Bonal, D. and O. MonTEuUis (1997): Ex vitro survival, rooting and initial development of in vitro rooted vs unrooted microshoots from juvenile and mature Tectona grandis genotypes. Silvae Genetica, 46(5), 301-306.

BoNGA, J. M. (1982): Vegetative propagation in relation to juvenility, maturity and rejuvenation. In: BoNGA, J. M. and DuRzan, D. J. (Eds), Tissue culture in forestry. Martinus Nijhoff/Dr W. Junk publishers, The Hague: 387-412.

Burdon, R. D. and C. J. A. Shelbourne (1974): The use of vegetative propagules for obtaining genetic information. N.Z.J. For. Sci. 4: 418-425.

Callister, A. N. and S. L. Collins (2008): Genetic parameter estimates in a clonally replicated progeny test of teak (Tectona grandis Linn.f.). Tree Genet. Genomes 4: 237-245.

Chaix, G., O. Monteuuis, C. Garcia, D. Alloysius, J. Gidiman, R. BACILIERI and D. K. S. GoH (2011): Genetic variation in major phenotypic traits among diverse genetic origins of teak (Tectona grandis L.f.) planted in Taliwas, Sabah, East Malaysia. Annals of Forest Science 68: 1015-1026.

Cheverud, J. M. (1988): A comparison of genetic and phenotypic correlations. Evolution 42(5): 958-968.

DANARTO, S. and E. B. HARDIYANTO (2001): Results of the progeny test of teak at 12 years of age at Jember, Esast
Java. In: "Potential and opportunities in marketing and trade of plantation teak: challenge for the new millenium". Proceeding of the Third Regional Seminar on Teak, Yogyakarta, Indonesia, 31 July-4 August 2000, 249-253.

FALCONER, D. S. (1974): Introduction à la génétique quantitative. Masson et Cie, Paris, 284p.

FALCONER, D. S. and F. C. MACKAY (1996): Introduction to quantitative genetics, $4^{\text {th }}$ ed. Longman, London, $457 \mathrm{p}$.

FAO (2009): The future of teak and the high-grade tropical hardwood sector: solving the Tropical Hardwood Crisis with Emphasis on teak (Tectona grandis Linn f.) Planted Forests and Trees Working Paper FP/44E, Rome, 37p. http://www.fao.org/forestry/site/10368/en/.

Frampton, J. and G. S. Foster (1993): Field testing vegetative propagules. In: Clonal forestry I. Genetic and biotechnology. AhUJA, M. H. and LiBBY, W. J. (eds). Springer-Verlag, Berlin, Heidelberg: 110-134.

Gezan, S. A., T. L. White and D. A. Huber (2006): Achieving higher heritabilities through improved design and analysis of clonal trials. Can J. For. Res. 36: 2148-2156.

Gogate, M. G., D. Gujar, A. K. Mandal, R. Sharma, R. B. LAL and B. N. GUPTA (1997): Genetic analysis of quantitative characters in teak (Tectona grandis). Ann. Forest Sci. 5(2): 165-167.

GoH, D. K. S. and O. MonteuUis (2005): Rationale for developing intensive teak clonal plantations, with special reference to Sabah. Bois et Forêts des Tropiques 285: $5-15$.

Goh, D. K. S., G. Chaix, H. Bailleres and O. MonteuUis (2007): Mass production and quality control of teak clones for tropical plantations: The Yayasan Sabah Group and Forestry Department of Cirad Joint Project as a case study. Bois et Forêts des Tropiques 293: $65-77$.

GoH, D. K. S. and O. MonteuUIS (2012): Behaviour of the "YSG BIOTECH TG1-8" teak clones under various site conditions: first observations. Bois et Forêts des Tropiques 311: 5-19.

HARShaP, R. M. S. and I. Soerianegara (1977): Heritability of some characters in teak (Tectona grandis L.f.) Third World Consultation on Forest Tree Breeding, Volume 2. CSIRO, Canberra, Australia: 649-657.

Hartmann, H. T., D. E. Kester, F. T. Davies and R. L. GENEVE (1997): Plant propagation: principles and practices, $6^{\text {th }}$ edition Englewood Cliffs, New Jersey, 770p.

KADAMBI, K. (1972): Silviculture and management of teak. Bulletin 24, Stephen F. Austin State University, Nacogdoches, Texas, USA 138p.

KAOSA-ARD, A. (2000): Gains from provenance selection. In: "Site, technology and productivity of teak plantations". FORSPA Publication $\mathrm{N}^{\circ}$ 24/2000, TEAKNET Publication $\mathrm{N}^{\circ}$ 3, 191-207.

KJAeR, E. D., E. B. LAURIDSEN and H. WELlENdORF (1995): Second evaluation of an international series of teak provenance trials. DANIDA Forest Seed Centre, Humlebaek, Arboretum, Horsholm. Denmark. 118p.

KJAER, E. D. and E. B. LAURIDSEN (1996): Results from a second evaluation of DFSC coordinated teak (Tectona grandis) provenance trials: has new information been obtained? In: Proc of Tree Improvement for sustainable tropical forestry, QFRI-IUFRO, Caloundra, Queensland, Australia, 27 October-1 November 1996, 154-157.

Kollert, W. and L. CHERUBINI (2012): Teak resources and market assessment 2010. FAO Planted Forests and Trees Working Paper FP/47/E, Rome, 42 p. 
LiBBy, W. J. (1974): The use of vegetative propagules in forest genetics and tree improvement. N.Z.J. For. Sci., 4: $440-447$.

LIBBY, W. J. and E. JUND (1962): Variance associated with cloning. Heredity, 17, 533-540.

Lothrop, J. E., R. E. ATKINS and O. S. SMith (1985): Variability for yield and yield components in IAP1R grain sorghum random-mating population. I. Means, variance components, and heritabilities. Crop Science 25: 235-240.

Madoffe, S. S. and J. A. MAGhembe (1988): Performance of teak (Tectona grandis L.f.) provenances seventeen years after planting at Longuza, Tanzania. Silv. Genet. 37, 5-6: 175-178.

MonteuUis, O. (1985): La multiplication végétative du séquoia géant en vue du clonage. Annales AFOCEL 1984, 139-171.

MonTEUUIS, O. (1995): Recent advances in clonal propagation of teak. In: Proc. of the International Workshop of BIO-REFOR, Kangar, Malaysia, Nov. 28-Dec. 1, 1994: 117-121.

Monteuuis, O., D. Vallauri, C. Poupard, L. Hazard, Y. Yusof, L. A. Wahap, C. Garcia and M. Chauvière (1995): Propagation clonale de tecks matures par bouturage horticole. Bois et Forêts des Tropiques 243: 25-39.

MonteuUis, O., M. C. Bon and D. K. S. GoH (1998): Teak propagation by in vitro culture. Bois et Forêts des Tropiques 256: $43-53$.

MonteuUis, O. and D. K. S. GoH (1999): About the use of clones in teak. Bois et Forêts des Tropiques 261: 28-38.

Monteuuis, O., D. K. S. Goh, C. Garcia, D. Alloysius, J. Gidiman, R. Bacilieri and G. ChaIX (2011): Genetic variation of growth and tree quality traits among 42 diverse genetic origins of Tectona grandis planted under humid tropical conditions in Sabah, East Malaysia. Tree Genet. Genomes 7: 1263-1275.

NA'IEM, M. (2001): Early performance of clonal test of teak. In: "Potential and opportunities in marketing and trade of plantation teak: challenge for the new millenium". Proceeding of the Third Regional Seminar on Teak, Yogyakarta, Indonesia, 31 July-4 August 2000: 271-275.

Olesen, P. O. (1978): On cyclophysis and topophysis. Silv. Genet. 27, 5: 173-17865.

Palanisamy, K., K. Gireesan, V. Nagarajan and M. Hegde (2009): Selection and clonal multiplication of superior trees of teak (Tectona grandis L.) and preliminary evaluation of clones. J. Tropical Forest Science, 21(2): 168-174.

SAS Institute Inc. (2008): SAS/STAT ${ }^{\circledR} 9.2$ User's Guide. Cary, NC: SAS Institute Inc.
SEARLE, S. R. (1961): Phenotypic, genotypic and environmental correlation. Biometrics, 17: 474-480. Phenotypic, genotypic and environmental correlations. Biometrics 17: 474-480.

SiswamartanA, S. (2000): Productivity of teak plantations in Indonesia. In: "Site, technology and productivity of teak plantations". FORSPA Publication $\mathrm{N}^{\circ} 24 / 2000$, TEAKNET Publication $\mathrm{N}^{\circ} 3,137-143$.

Siswamartana, S. and A. Wibowo (2005): Early performance clonal tests of teak in Perum Perhutani Java. Paper presented at the $22^{\text {nd }}$ IUFRO World Congress Forests in balance: linking tradition and technology, 8-13 August 2005, Brisbane, Australia: 70-77.

Smit, L., L. A. Ugalde, A. P. DA Silva and E. O. Filho (2011): Plantation performance of teak clones and seeds in different types of soils at 5.2 years of age in Tangara de la Serra, Mato Grosso, Brazil. http://web.catie.ac.cr/ conferencia_teca/presentaciones/Session_7_7.pdf.

SoKAL, R. R. and J. F. ROHLF (1995): Biometry. $3^{\text {rd }}$ Ed., W. H. Freeman and Company, New York, USA, 887p.

SolórZano NARANJo, S., R. MoYa and S. Chauhan (2012): Early genetic evaluation of morphology and some wood properties of Tectona grandis L. clones. Silv. Genet. 61, 1-2: 58-65.

Suksileung, P., L. Chuntanaparb, C. Khemnark and S. SRIWATANAPONE (1975): Clonal variation and inheritance in growth characteristics of teak (Tectona grandis L.). The Kasersart Journal 9: 1-11.

TEWARI, D. N. (1992): A monograph on teak (Tectona grandis Linn. f.). International book distributors, Dehra Dun, India, 479p.

Timmis, M. M., Abo EL-Nil and R. W. Stonecypher (1987): Potential genetic gain through tissue culture. In: Cell and Tissue Culture in Forestry, J. M. BongA, D. J. DuRZAN (eds). Dordrecht, Germany, Martinus Nijhoff Publishers, 198-215.

Verhaegen, D., I. J. Fofana, Z. A. Logossa and D. Ofori (2010): What is the genetic origin of teak (Tectona grandis L.) introduced in Africa and Indonesia? Tree Genet. Genomes 6(5): 717-733.

WARDANI, B. W., M. NA'IEM and E. B. HaRdiyanto (2009): Genetic Parameter Estimates for Growth and Stem Form in a 9-Year Old Clonal Tests of Teak (Tectona grandis Linn. f.) in Java. Paper presented at the second Australasian Forest Genetics Conference, 20-22 Apr 2009, Perth, Australia, 8p.

White, T. L., W. T. Adams and D. B. Neale (2007): Forest Genetics. Cabi publishing, Oxfordshire, Cambridge, $682 \mathrm{p}$.

Zobel, B. and J. TAlBert (1984): Applied Forest Tree Improvement. John Wiley \& Sons, New York, Chichester, Brisbane, Toronto, Singapore, 505p. 